\title{
Efficient genome editing in filamentous fungus Trichoderma reesei using the CRISPR/Cas9 system
}

\author{
Rui Liu*, Ling Chen*, Yanping Jiang, Zhihua Zhou, Gen Zou \\ CAS-Key Laboratory of Synthetic Biology, Institute of Plant Physiology and Ecology, Shanghai Institutes for Biological \\ Sciences, Chinese Academy of Sciences, Shanghai, China
}

Filamentous fungi have wide applications in biotechnology. The CRISPR/Cas9 system is a powerful genome-editing method that facilitates genetic alterations of genomes in a variety of organisms. However, a genome-editing approach has not been reported in filamentous fungi. Here, we demonstrated the establishment of a CRISPR/Cas9 system in the filamentous fungus Trichoderma reesei by specific codon optimization and in vitro RNA transcription. It was shown that the CRISPR/Cas9 system was controllable and conditional through inducible Cas9 expression. This system generated site-specific mutations in target genes through efficient homologous recombination, even using short homology arms. This system also provided an applicable and promising approach to targeting multiple genes simultaneously. Our results illustrate that the CRISPR/Cas9 system is a powerful genome-manipulating tool for T. reesei and most likely for other filamentous fungal species, which may accelerate studies on functional genomics and strain improvement in these filamentous fungi.

Keywords: genome-editing; CRISPR/Cas9; filamentous fungus; Trichoderma reesei

Cell Discovery (2015) 1, 15007; doi:10.1038/celldisc.2015.7; published online 12 May 2015

\section{Introduction}

Filamentous fungi, such as Trichoderma reesei, Aspergillus niger, A. oryzae, and Penicillium chrysogenum, have been used to produce diverse enzymes or metabolites for centuries [1]. To obtain hyper-producers, repeated classical mutagenesis and screening have been applied for long-term strain improvements. Genetic engineering approaches for filamentous fungi have been well developed. However, these approaches are not as efficient as those available for yeast and bacteria due to the additional complexity of filamentous fungi [2], such as multicellular morphology, cellular differentiation, thick chitinous cell walls, and the lack of suitable plasmids.

*These two authors contributed equally to this work.

Correspondence: Zhihua Zhou

Tel: +86 215492 4050; Fax: +862154924049

E-mail: zhouzhihua@sippe.ac.cn

or Gen Zou

Tel: +86 215492 4049; Fax: +86 2154924049

E-mail: zougen@sibs.ac.cn

Received 28 December 2014; accepted 28 February 2015
Genome-editing is a type of genetic engineering in which DNA is inserted, replaced, or removed from a genome using artificially engineered nucleases, or 'molecular scissors'. It is a useful tool to elucidate the function and effect of a gene or protein in a sequencespecific manner [3]. Recently, developed engineering nucleases, zinc-finger nucleases [4] and transcription activator-like effector nucleases [5], are believed to facilitate precise genome modifications. However, the time- and labor-intensive process of using zinc-finger nucleases and the requirements of specific enzyme engineering for different targets in transcription activator-like effector nucleases limit their applications. The type II clustered regularly interspaced short palindromic repeats (CRISPR)/CRISPR-associated gene (Cas) system is the most popular genome-editing tool at this time. A single multidomain Cas9 catalyzes a double-strand break (DSB) in the target DNA composed of a 20-bp sequence matching the protospacer of the guide RNA (gRNA) and an adjacent downstream 5 '-NGG nucleotide sequence (termed as the protospacer-adjacent motif (PAM)) [6]. Over the past 2 years, many studies have demonstrated that the 
CRISPR/Cas9 system is a powerful genome-editing method that facilitates genetic alterations in genomes in a variety of organisms. Until now, there have been no reports on the CRISPR/Cas9 system or other genome-editing approaches in filamentous fungi, even in the model organism Neurospora crassa, despite the successful application of this technique in yeast $[2,7]$.

The filamentous fungus $T$. reesei, which is recognized by its Generally Recognized as Safe status by the US Food and Drug Administration, is the most widely used producer of commercial lignocellulolytic enzyme preparations [1] and is expected to be a potential cell factory for different heterologous proteins with its powerful ability to synthesize and secrete proteins with high quantities. However, the genetic basis of desired domestication traits is poorly understood, because robust genetic tools do not exist for this industrial host. The establishment of a genome-editing system can be used to develop T. reesei as a super cell factory for lignocellulolytic enzyme preparations and other heterologous proteins, as well as to characterize the regulatory mechanisms for induction, synthesis, and secretion of proteins in $T$. reesei.

In this study, we constructed a CRISPR/Cas9 system in $T$. reesei that allowed us to induce mutagenesis or introduce a new gene by homologous recombination into a target site of the $T$. reesei genome. In addition, it can be used to generate multiple genome modifications simultaneously by co-transforming gRNAs and donor DNAs (dDNAs) for different targets. This system demonstrates great potential as a tool for genome engineering in filamentous fungi, owing to userdesignated site specificity of Cas9 endonuclease activity and the simplicity of gRNA construction.

\section{Results}

Expression of codon-optimized Cas 9 in T. reesei

Construction of the CRISPR/Cas9 system in an organism requires successful expression of Cas9. Unlike in Saccharomyces cerevisiae [7], the codonoptimized $\operatorname{Cas} 9$ gene for human cells does not function in T. reesei Qm6a or Rut-C30 under control of the constitutive promoter Ppdc (the promoter of $p d c$, the gene encoding pyruvate decarboxylase, which participates in glucose metabolism [8]) or the inducible promoter Pcbh1 (the promoter of $c b h 1$, the gene encoding cellobiohydrolase I, which is repressed by glucose but induced by a series of oligosaccharides or cellulose [9]). On the basis of the codon frequency of $T$. reesei (http://www.kazusa.or.jp/codon/cgi-bin/showcodon. cgi?species $=51453$ ), we optimized the $\operatorname{Cas} 9$ gene of Streptococcus pyogenes and the sequence of the SV40 nuclear localization signal (Supplementary Data S1). To detect the expression and localization of the optimized Cas9, the enhanced green fluorescent protein (eGFP) gene was fused to to Cas9 (optimized Cas 9 gene) and expressed in both the wild-type strain Qm6a and the mutant strain Rut-C30 under control of the Ppdc and Pcbh1 promoter, respectively (Supplementary a

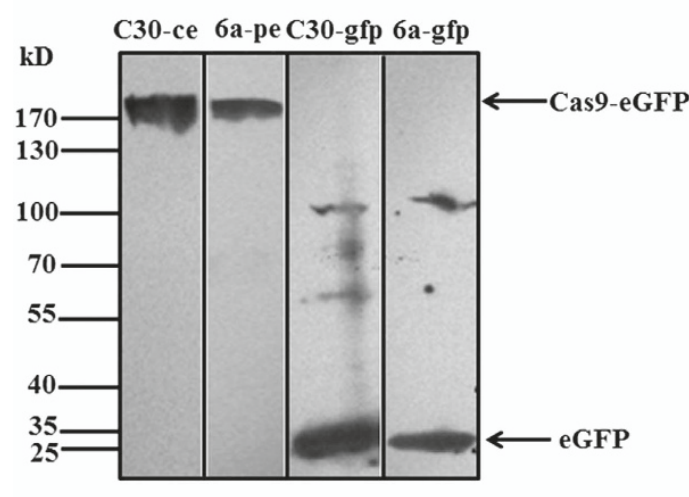

b

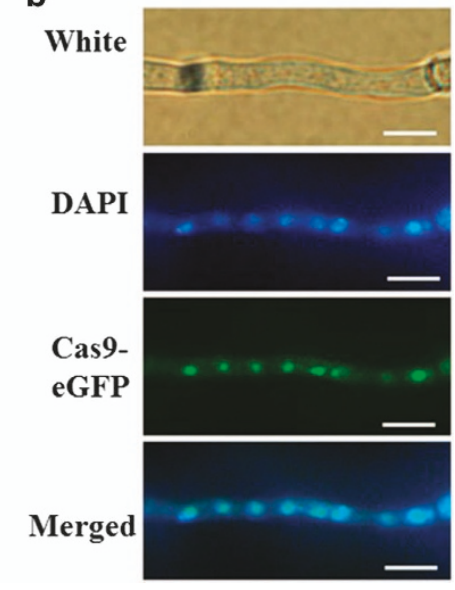

Figure 1 Expression and localization of Cas9-eGFP in T. reesei strains. (a) Detection of Cas9-eGFP expression in transformants 6a-pe and C30-ce by western blots. Transformants 6a-gfp and C30-gfp expressing eGFP were used as controls. Lanes C30-ce and 6a-pe were loaded with $0.05 \mathrm{mg}$ protein. Lanes C30-gfp and 6a-gfp were loaded only with $0.01 \mathrm{mg}$ protein to avoid overexposure. Anti-GFP antibody (Genscript, Nanjing, China) was used for detection. (b) Fluorescence microscopic assessment of the localization of Cas9-eGFP in C30-ce. The bars were $5 \mu \mathrm{m}$. DAPI, 4',6-diamidino-2-phenylindole; eGFP, enhanced green fluorescent protein. 
Data S1). The expression of toCas 9 in each of the selected transformants was detected based on western blots (Figure 1a). to $\operatorname{Cas} 9$ was expressed in both the transformant 6a-pe (introduced Ppdc-toCas9-eGFPTpde into Qm6a) and the transformant C30-ce (introduced Pcbh1-toCas9-eGFP-Tpdc into Rut-C30).

To determine whether the SV40 nuclear localization signal localizes Cas9 to the nucleus, $\mathrm{C} 30$-ce was assessed using fluorescence microscopy (Figure 1b). The mycelia of $\mathrm{C} 30$-ce glowed with punctiform green fluorescence after 2 days of growth on the inducing culture media containing 1\% Avicel. After staining with 4',6-diamidino-2-phenylindole $\left(1 \mu \mathrm{g} \mathrm{ml}^{-1}\right)$, the majority of the green fluorescence spots overlapped with nuclei (blue fluorescence). This demonstrated that Cas9 was located mostly in the nucleus of $T$. reesei, where Cas9 is believed to generate DSBs within the target gene.

To build Cas9-expressing chassis, the Ppdc-toCas9Tpdc cassette and Pcbh1-toCas9-Tpdc cassette were introduced into Qm6a and Rut-C30, respectively. To choose the Cas9 expression chassis for further investigations, we examined the growth and cellulase activities of the selected transformant 6a-pc from Qm6a and C30-cc from Rut-C30 after three consecutive subcultures for genetic stability, which did not show any differences compared with those of the parent strains (Supplementary Figure S1).

\section{CRISPR/Cas9 system directed URA5 mutagenesis in $T$. reesei}

Using appropriately designed gRNAs, the genome can be cut at any desired location by the Cas9 protein. Owing to the lack of confirmed RNA polymerase IIIbased promoters in $T$. reesei, such as the U6 promoter in Arabidopsis thaliana [10] and SNR52 promoter in $S$. cerevisiae [7], we preferred to introduce gRNAs into cells after transcription in vitro [11]. As ura5 has undergone negative selection, its inactivation can be observed in 5-FOA-containing minimal medium (MM) plates (cells expressing wild-type ura5 convert 5-FOA into the toxic substance $5^{\prime}$ fluorouridine monophosphate, which severely limits cell growth). Thus, gRNA was designed to target ura5 and was delivered into Cas9-expressing $T$. reesei cells through protoplast transformation. To ensure that Cas9 is active in cells, we first chose transformant 6a-pc (a transformant constitutively expressing Cas9) as the Cas9-expressing chassis to examine the mutation frequency. The ura5 genes of 14 selected 5-FOA-resistant transformants were sequenced to confirm whether the mutations were directed by CRISPR/Cas9 (Table 1). Each ura5 gene of the 14 selected 6a-pc transformants showed a frameshift mutation at the expected target site, proximally located upstream of the PAM sequence (Figure 2a). This was consistent with the fact that CRISPR/Cas9guided DSBs can be repaired through nonhomologous end joining (NHEJ), which generates insertions and deletions in the vicinity of the cleavage site. By contrast, no colony was observed in 5-FOA-containing MM plates from the control transformation of 6a-pc without gRNA. It confirmed that gRNA might be the essential component to result in mutations.

Controllability of the CRISPR/Cas9 system in T. reesei

In industries, the majority of filamentous fungi produce metabolites or enzymes at a specific stage or under unique conditions. A spatiotemporally controllable genome-editing tool precisely controls the metabolic shift in industry bioprocesses. We tested the conditionality of CRISPR/Cas9-guided mutagenesis in the Cas9-expressing chassis, C30-cc (a transformant expressing Cas9 only under inducing conditions) (Supplementary Data S1). As expected, mutagenesis was performed conditionally by an inducer (such as lactose or cellulose) in C30-cc. In the inducible medium (lactose or cellulose as the sole carbon resource), all 15 selected transformants (AR1-AR15) showed mutations in the target gene (Figure 2a). However, in the repressing medium (containing glucose, a repressor for promoter Pcbh1 that controls the $\operatorname{Cas} 9$ gene expression), none of the colonies showed mutations. Besides, no colony was observed in 5-FOAcontaining MM plates from the control transformation of 6a-pc without gRNA. The above results demonstrated that the CRISPR/Cas9 system functions in $T$. reesei as an efficient and controllable genome-editing tool in a sequence-specific manner. Besides, the expression of Cas9 under an inducible promoter or other conditionally activated promoters renders the CRISPR/Cas9 system a spatiotemporal-controller of genome-editing of filamentous fungi.

\section{CRISPR/Cas9 system stimulated homologous recombination in $T$. reesei}

Similarly to inducing mutagenesis in a sequencespecific manner, the CRISPR/Cas9 system also induces homologous recombination between endogenous and exogenous DNAs. We used poura5 (an exogenous ura5 gene, encoding URA5 in $P$. oxalicum [12]) as a selectable marker and the endogenous putative methyltranferase lael gene as a target to test the incidence rate of homologous recombination (Figure 2b) by the CRISPR/Cas9 system [13]. The uridine- 
Table 1 Primers and oligonucleotides used in this study

\begin{tabular}{|c|c|c|}
\hline Name & Sequence (5' to $\left.3^{\prime}\right)$ & Experiment \\
\hline ToCas9F & AGCGCAGCTACAGCACAATCATGGACAAGAAGTACAGCAT & Cloning of tocas 9 \\
\hline ToCas9R & TTAGACCTTGCGCTTCTTCTTGGGGTCGGCGCGGGAGTCG & Cloning of toCas 9 \\
\hline PpdcF & ACGACGGCCAGTGCCAAGCTTAGGACTTCCAGGGCTACTTG & Cloning of $p d c$ promoter \\
\hline PpdcR & GATTGTGCTGTAGCTGCGCTGCTTTGATCGTTTTGAGGTGC & Cloning of $p d c$ promoter \\
\hline pcbhlcasF & ACGACGGCCAGTGCCAAGCTTTTTCCCTGATTCAGCGTACC & Cloning of $c b h 1$ promoter \\
\hline pcbh1casR & TTGACTATTGGGTTTCTGTGCCTC & Cloning of $c b h 1$ promoter \\
\hline eGFPF & GTGGAGACAGCAGGGCTGACGTACCGGTCGCCACCATGGTG & Cloning of $e G F P$ \\
\hline eGFPR & TTACACСТTCСТСТTCTTCTTGGGCTTGTACAGCTCGTCCATG & Cloning of $e G F P$ \\
\hline TpdcF & AGAAGAAGAGGAAGGTGTGACCCGGCATGAAGTCTGACCG & Cloning of $p d c$ terminator \\
\hline TpdcR & TAATTGCGCGGATCCTCTAGATGGACGCCTCGATGTCTTCC & Cloning of $p d c$ terminator \\
\hline ToCGFPF & GCGGCGACTCCCGCGCCGACGTACCGGTCGCCACCATGGTG & Overlapping $e G F P$ to to Cas 9 \\
\hline GFPTrCasR & GTCGGCGCGGGAGTCGCCGC & Overlapping eGFP to to Cas 9 \\
\hline lae1 up F & TGCAGGTCGACGATTGAAGCCAATCAGCAAGGTTGAACTG & Cloning the $5^{\prime}$ flanking region of lael \\
\hline lael up $\mathrm{R}$ & TACTGGCTTAACTATGCGGCTTGGCATACCTGAAAAATGT & Cloning the $5^{\prime}$ flanking region of lael \\
\hline lae1-0.2 F & TGCAGGTCGACGATTATGTCTTTCACAAGTATTTC & $\begin{array}{l}\text { Cloning the } 5^{\prime} \text { flanking region }(0.2 \mathrm{~kb}) \\
\text { of lae } 1\end{array}$ \\
\hline lae1-0.4 F & TGCAGGTCGACGATTAACCGGTCAGCGAGCC & $\begin{array}{l}\text { Cloning the } 5^{\prime} \text { flanking region }(0.4 \mathrm{~kb}) \\
\text { of lae } 1\end{array}$ \\
\hline lae1-0.6 F & TGCAGGTCGACGATTGCACTGGACTCTGGAAC & $\begin{array}{l}\text { Cloning the } 5^{\prime} \text { flanking region }(0.6 \mathrm{~kb}) \\
\text { of lae } 1\end{array}$ \\
\hline lae1-0.8 F & TGCAGGTCGACGATTCGTTATACTGTACTTAACAAG & $\begin{array}{l}\text { Cloning the } 5^{\prime} \text { flanking region }(0.8 \mathrm{~kb}) \\
\text { of lae1 }\end{array}$ \\
\hline THRUra5 F & GCCGCATAGTTAAGCCAGTATACACTCCGCTATCGCTACG & $\begin{array}{l}\text { Cloning the } 5^{\prime} \text { flanking region of } \\
\text { donor DNA }\end{array}$ \\
\hline THRUra5 R & CAGGGCTGGTGACGGAATTTTCATAGTCAAGCTATCAGAG & $\begin{array}{l}\text { Cloning the } 5^{\prime} \text { flanking region of } \\
\text { donor DNA }\end{array}$ \\
\hline lae1 down $\mathrm{F}$ & AAATTCCGTCACCAGCCCTGCGGCAGCATAAGAGATGACAA & Cloning the $3^{\prime}$ flanking region of lael \\
\hline lael down $\mathrm{R}$ & GGATCCTCTAGAGATGTGCTTCATTCGAATGAGACAGAGC & Cloning the $3^{\prime}$ flanking region of lael \\
\hline lae1-0.2 R & GGATCCTCTAGAGATTGAAGAGCCGAATGTCTG & $\begin{array}{l}\text { Cloning the } 3^{\prime} \text { flanking region }(0.2 \mathrm{~kb}) \\
\text { of lae } 1\end{array}$ \\
\hline lae1-0.4 R & GGATCCTCTAGAGATGGCTGAAGGCGAGGTTGAAC & $\begin{array}{l}\text { Cloning the } 3^{\prime} \text { flanking region }(0.4 \mathrm{~kb}) \\
\text { of lae } 1\end{array}$ \\
\hline lae1-0.6 R & GGATCCTCTAGAGATAAATGTGTCTGCAAGCGTGATTAG & $\begin{array}{l}\text { Cloning the } 3^{\prime} \text { flanking region }(0.6 \mathrm{~kb}) \\
\text { of lae } 1\end{array}$ \\
\hline lae1-0.8 R & GGATCCTCTAGAGATCCGCAGAACGCTGGCTCTC & $\begin{array}{l}\text { Cloning the } 3^{\prime} \text { flanking region }(0.8 \mathrm{~kb}) \\
\text { of lael }\end{array}$ \\
\hline Ulae1 F & GGCAACCCACCCGTACCACA & Verifying the $5^{\prime}$ flanking region of lael \\
\hline HR ura5 R & TGCTCGATGATCCCGACAGC & $\begin{array}{l}\text { Cloning the } 3^{\prime} \text { flanking region of } \\
\text { poura } 5 \text { cassette }\end{array}$ \\
\hline HR ura5 F & AATCGCCCTACTTCTTCACA & $\begin{array}{l}\text { Cloning the } 5^{\prime} \text { flanking region of } \\
\text { poura } 5 \text { cassette }\end{array}$ \\
\hline Dlae1 R & CAGGCTCTTATCGCCATCTC & Verifying the $3^{\prime}$ flanking region of lael \\
\hline Tura5 F & GCGGCGTCCTCAAGTTTGGC & Sequencing and verifying ura5 \\
\hline Tura5 R & CGGTAATCCTCCGTGTTCTT & Sequencing and verifying ura5 \\
\hline
\end{tabular}




\section{a}

\begin{tabular}{|c|c|}
\hline $\begin{array}{l}\text { Qm6a } \\
\text { Tura5 }\end{array}$ & ССТACTCGTTTGACCGCAAGGAGGCCAAGGACCACGGCGAGGGCGGCAACAT--CGTCGGCGCTTCGCTCAAGGGCAAGAGGGTCCTGATTGTCGACGACGTCATCACCGCCGGCACCGC \\
\hline & CCTACTCGTTTGACCGCAAGGA GGCCAAGGA CCACGGCGAGGGCGGCAACA---CGTCGGCGCTTCGCTCAAGGGCAAGAGGGTCCTGA TTGTCGA CGA CGTCA TCACCGCCGGCACCGC \\
\hline & CCTACTCGTTTGACCGCAAGGAGGCCAAGGACCACGGCGAGGGCGGCAACATA-CGTCGGCGCTTCGCTCAAGGGCAAGAGGGTCCTGA TTGTCGACGACGTCA TCACCGCCGGCACCGC \\
\hline & CCTACTCGTTTGACCGCAAGGAGGCCAAGGACCACGGCGAGGGCGG- \\
\hline & CTCAAGGGCAAGAGGGTCCTGATTGTCGACGACGTCATCACCGCCGGCACCGC \\
\hline & TCGTTTGACCGCAAGGAGGCCAAGGACCACGGCGAGGGCGGC \\
\hline & TACTCGTTTGACCGCAAGGAGGCCAAGGACCACGGCGAGGGCGGCA \\
\hline & GCTCAAGGGCAAGAGGGTCCTGATTGTCGACGACGTCATCACCGCCGGCACCGC \\
\hline ( & CCTACTCGTTTGACCGCAAGGAGGCCAAGGACCACGGCGAGGGCGGCAA \\
\hline & CAAGAGGGTCCTGATTGTCGACGACGT \\
\hline 10 & GGCAAGAGGGTCCTGATTGTCGACGACGTC \\
\hline 11 & CGCCGGCACCGC \\
\hline 12 & TCACCGCCGGC \\
\hline 13 & GAGGGCGGC \\
\hline 14 & TТ СТCCTTTTCACCCCAACCA CCCCAACCACCA CCCCCA CCCCCCO \\
\hline \multicolumn{2}{|l|}{ Rut- } \\
\hline & \\
\hline & CCT $A$ + \\
\hline & CCTACTCGTTTGACCGCAAGGAGGCCAAGGACCACGGCGAGGGC \\
\hline & CCGC \\
\hline & ACCACGC \\
\hline & 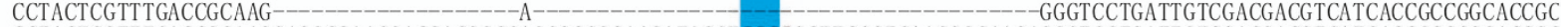 \\
\hline & GTTTGACCGCA \\
\hline 8 & $\mathrm{CC}$ \\
\hline & \\
\hline 10 & \\
\hline 11 & CGCCGGCACCG \\
\hline 12 & \\
\hline 13 & CGC \\
\hline 14 & 10 (1) \\
\hline 10 & \\
\hline
\end{tabular}

b
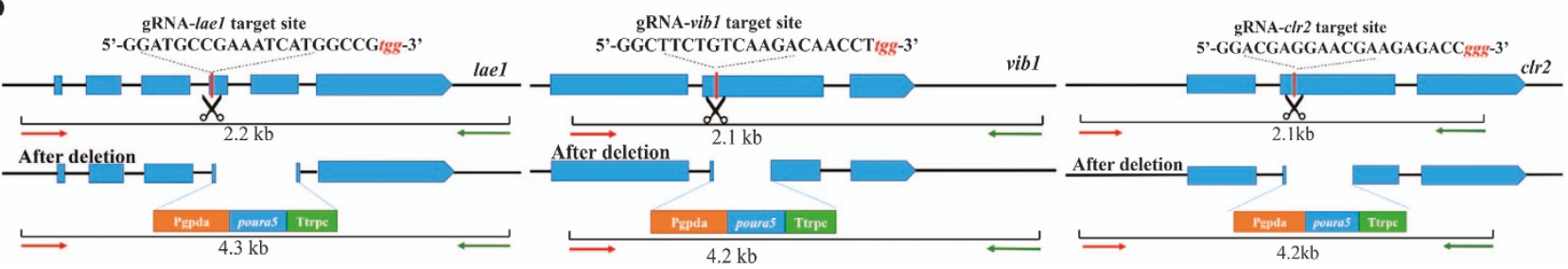

Figure 2 CRISPR/Cas9-based mutagenesis and homologous recombination in T. reesei. (a) Alignments of ura5 mutants from 5-FOA-resistant colonies constitutive expression or post-lactose induction (the gene encoding Cas9 under control of Pcbh1 can be induced by oligosaccharides such as lactose, sophorose, or cellodextrin, in C30-cc) of Cas9 in gRNA transformants (14 6a-pc transformants and $15 \mathrm{C} 30$-cc transformants). Tuar5 is the wild-type reference ura5 gene from T. reesei Qm6a and Rut-C30 (the PAM sequence is highlighted in blue and the guide RNA (gRNA)-guiding sequence in green). Deletions or insertions were found near the PAM sequence. (b) Schematic for homologous recombination (HR) of lae1, vib1, and clr2 mediated by Cas9 and donor DNAs. Results of PCR analyses revealed HR events.

dependent transformant AR3 (derived from chassis C30-cc, Figure 2a) was used as a host. After cotransformation of gRNA (gRNA-lae1, targeting lae1 site) with dDNA (dDNA-lael, containing Pgpdapoura5-Ttrpc cassette and the $3^{\prime}$ and $5^{\prime}$ flanking regions of lae1) (Table 1), the transformants could be selected in MM plates. The sequence of the selectable marker cassette replaced lael in all 14 transformants (Supplementary Figure S2). All slael transformants showed weakened sporulation (Figure $3 a$ ) and cellulolytic capability (Figure 3b) compared with the controls, as expected [13]. Compared with other genetic approaches reportedly used for homologous recombination in filamentous fungi [14], the efficiency and precision of the CRISPR/Cas9 system supported its significant advantages.
The effect of the length of homology arms on the recombination frequency was also investigated. The uridine-dependent Cas9 expression chassis AR3 was transformed with gRNA-lael and a set of dDNAs with various lengths of homology arms ranging from 0.2 to $1.0 \mathrm{~kb}$ at $0.2-\mathrm{kb}$ intervals (dDNA-lae1-0.2k, dDNA-lae1-0.4k, dDNA-lae1-0.6k, dDNA-lae1-0.8k and dDNA-lae1) (Supplementary Data S1). Nine, 14 or 15 transformants (monoconidial cultures) from each transformation were selected and then checked using diagnostic PCR. These results are summarized in Table 2. The homologous recombination frequencies for all tested homology arms were $\geqslant 93 \%$. These results demonstrated that a pair of 200-bp homology arms is sufficient to achieve efficient homologous integration stimulated by the CRISPR/Cas9 system in $T$. reesei. 
a

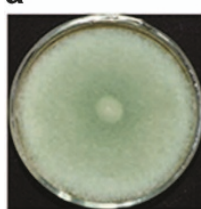

Rut-C30

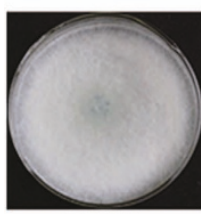

$\Delta$ lael-1

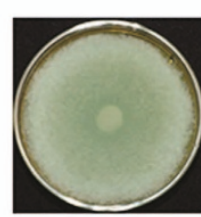

C30-cc

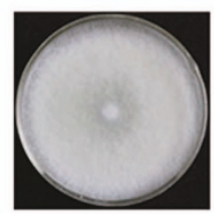

$\Delta$ lael-2

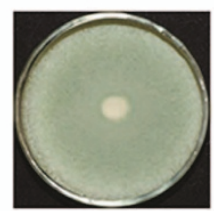

AR3

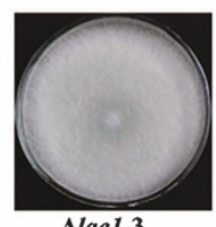

b

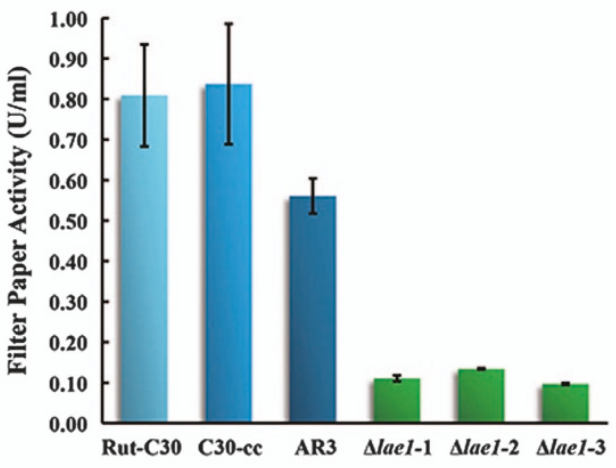

Figure 3 Reduction of sporulation and cellulolytic capability in $\Delta / a e 1$ strains. (a) The observed sporulation of mutants and controls in potato dextrose agar plates after 7 days of growth. Sporulation was reduced in all three randomly selected $\Delta / a e 1$ strains compared with controls. (b) Assays for filter paper activity of $\Delta / a e 1$ strains and controls in inducing medium with $1 \%$ lactose after 3 days of fermentation. The cellulolytic capability was weakened significantly $(P<0.01, t$-test). Error bars represent the s.d. among three independent measurements.

Table 2 Homologous recombination frequencies at the lae1 locus using homology arms of various lengths

\begin{tabular}{lccc}
\hline $\begin{array}{l}\text { Homology } \\
\text { arm size (kb) }\end{array}$ & $\begin{array}{c}\text { No. of analyzed } \\
\text { transformants }\end{array}$ & $\begin{array}{c}\text { No. of } \\
\text { deletants }\end{array}$ & $\begin{array}{c}\text { Homologous } \\
\text { recombination } \\
\text { frequency (\%) }\end{array}$ \\
\hline 0.2 & 15 & 14 & 93 \\
0.4 & 15 & 14 & 93 \\
0.6 & 15 & 15 & 100 \\
0.8 & 9 & 9 & 100 \\
1.0 & 14 & 14 & 100 \\
\hline
\end{tabular}

Simultaneous homologous recombination of multigenes using the CRISPR/Cas9 system in T. reesei

Disruptions or homologous recombinations of multigenes are required during strain modification or to investigate regulatory systems for a unique metabolic process in filamentous fungi. A genetic approach that could target multiple genes simultaneously would greatly benefit such studies. In the uridine-dependent Cas9 expression chassis AR3, we tested the efficiency of multiple genome modifications directed by the CRISPR/Cas9 system. The selectable marker (Pgpdapoura5-Ttrpc cassette) was used to simultaneously target lael and vib1 (coding VIB1, a putative link between glucose signaling and carbon catabolite repression [15]). The gRNA and dDNA targeting vibl were termed gRNA-vibl and dDNA-vibl, respectively (containing Pgpda-poura5-Ttrpc cassette and the $3^{\prime}$ and $5^{\prime}$ flanking regions of vibl)
(Supplementary Data S1). After co-transformation of the two sets of gRNA and dDNA at the same molar concentration (gRNA-vibl:dDNA-vibl:gRNA-lael: dDNA-lae1 =1:1:1:1), 24 transformants were selected in the uridine-free medium. All 18 positive transformants integrated the selectable marker cassette into the lael locus. However, only three showed homologous recombination in both lael and vibl loci. The frequency for double recombination was only $16 \%$ (Supplementary Figure S3).

As all the single-deletion transformants were $\Delta$ lael strains, the molar concentration ratio of the gRNA and dDNA targeting vibl to those targeting lael was adjusted to 1.5:1:1:1 (gRNA-vib1:dDNA-vib1:gRNAlae1:dDNA-lae1) in the next co-transformation test. Eleven of the 24 randomly selected transformants were double-deletion strains. The frequency of double recombination was $\sim 45 \%$, and the remaining strains were all single-lael deletion transformants (Supplementary Figure S3).

To test the efficiency of simultaneous homologous recombination of triple genes, clr2 used as the third target, which is a conserved and essential transcription factor for cellulase gene expression in ascomycete fungi [16]. After co-transformation of the three sets of gRNA and dDNA at the same molar concentration (gRNA-lae1:dDNA-lae1:gRNA-vib1:dDNA-vib1:

gRNA-clr2:dDNA-clr2 =1:1:1:1:1:1), 24 transformants were selected in uridine-free medium, in which there was one triple-gene deletion $(4.2 \%)$, seven $\Delta$ lae1 $\Delta$ clr 2 double deletions, 13 slael single deletions, and two $\Delta c l r 2$ single deletions (Supplementary Figure 
S4). Although the frequency of triple recombination was quite low, the CRISPR/Cas9 system is an applicable and promising approach to target multiple genes simultaneously.

\section{Discussion}

High-efficiency genetic manipulation approaches facilitate fungal strain improvement, as well as the elucidation of fungal molecular mechanisms behind the industrial applications. The first reports of genetic transformation in Aspergillus were in 1983 [17, 18], which lagged behind those for Escherichia coli and $S$. cerevisiae. Over the past three decades, scientists have attempted to develop various tools or strategies to improve genetic manipulation approaches in filamentous fungi [19-21]. Different genetic transformation approaches have been applied for homologous recombination in many filamentous fungi; however, the low homologous recombination frequency $(<5 \%)$ has limited studies on their functional genomics through targeted gene replacement [22]. Deletion of the $\mathrm{Ku} 70$ or $\mathrm{Ku} 80$ heterodimer (Ku complex) increased the frequency of homologous recombination by up to $65 \%$ in many filamentous fungi by eliminating the NHEJ pathway [23]. However, $\Delta k u$ strains were more sensitive to growth environments with specific chemicals such as phleomycin, bleomycin, and methyl/ethyl methanesulfonate. Besides, the strategy to delete the $\mathrm{Ku} 70$ or $\mathrm{Ku} 80$ heterodimer is not suitable for Agrobacteriummediated fungal transformation because of its incompatibility in NHEJ-deficient strains [24]. In this study, the CRISPR/Cas9 system showed the highest frequency of single homologous recombination in $T$. reesei (almost $100 \%$ ) using a pair of $\geqslant 600$-bp homology arms (Table 2). Even when the length of the homology arms was decreased to $200 \mathrm{bp}$, the CRISPR/Cas9 system still reached a $93 \%$ homologous recombination frequency (Table 2), higher than the frequency in NHEJ-deficient T. reesei using 500-bp homology arms (63\%) [25].

Establishing the CRISPR/Cas9 system in filamentous fungi is based on basic transformation systems. High frequencies of multicopy insertion and ectopic integration have been observed in the application of these filamentous fungal transformation systems $[25,26]$. Compared with $\mathrm{PEG} / \mathrm{CaCl}_{2}$ protoplast, electroporation and biolistic transformation systems, A. tumefaciens-mediated transformation alleviates variable multicopy insertion and ectopic integration with its high efficiency $(>90 \%)$ of single-copy integration in $T$. reesei [27]. In this study, we introduced the Cas9-encoding gene into the genome of $T$. reesei using random A. tumefaciens-mediated transformation. To ensure that the introduction and expression of the Cas9-encoding gene does not disturb the expression and function of other genes, the morphology and important phenotypes such as growth and cellulase activities of the selected transformants were compared with their parent strains (Supplementary Figure S1). Although the confirmation process for Cas9 expression typically takes more than 1 month, similarly to previous transformation systems, the Cas9 expression chassis (including the uridine-dependent Cas9 expression chassis) could be used in the following studies for different gene mutations or gene knockout /knock-in by changing the 20-bp protospacer of gRNA to the target locus according to the fungal genomic sequence information. Thus, the CRISPR/Cas9 system in filamentous fungi would save time and labor in the study of fungal life activity regulatory systems, such as reproduction, development, metabolic pathways, protein synthesis, and secretion, which involve multiple unknown functional genes. For example, it took more than a decade to construct genome-wide single-gene deletion libraries for functional genomics research in $N$. crassa and $A$. nidulans using traditional genetic manipulation approaches [28, 29]. The CRISPR/Cas9 system is expected to shorten the process for establishing a genome-wide single-gene deletion library for a filamentous fungal species with available genomic sequence information.

Owing to the site specificity of the Cas9 endonuclease and gRNA, the CRISPR/Cas9 system is believed to introduce a genetic alteration or a heterologous gene into the target locus precisely and efficiently. In this study, variations in the target ura5 gene were detected in all selected mutagenesis transformants (Figure 2a), and poura 5 was shown to replace the target gene lael in all homologous recombination transformants (Supplementary Figure S2). Even in the screened homologous recombination transformants simultaneously targeting triple loci, the heterologous gene poura5 was shown to replace target genes lae1, vib1, and clr2 or at least one or two of these genes (Supplementary Figure S4). Our results confirmed that the CRISPR/Cas9 system was successful in editing the genome of a filamentous fungus precisely and efficiently, similarly to other organisms.

In our simultaneous homologous recombination experiments targeting double genes, almost all of the selected single-deletion transformants were slael strains (Supplementary Figure S3). We did not obtain a $\Delta$ vibl strain, and DSBs were not observed in the gRNA-directed vibl locus. Similarly, in the 
simultaneous homologous recombination experiments targeting triple genes, the selected single-deletion transformants were $\Delta$ lael or $\Delta$ clr 2 strains, and the selected double deletion was $\Delta$ lae $1 / \Delta c l r 2$. No $\Delta v i b 1$,

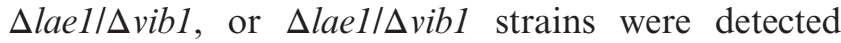
among the selected transformants (Supplementary Figure S4). The CRISPR/Cas9 system prefers to target the lael or clr2 locus rather than the vib1 locus. The gene vibl may be located in a region of low transcriptional activity [30], which complicates the process of gRNA directing Cas9 to the vibl locus. The increase in the molar concentration ratio of gRNA-vibl (the gRNA targeting vibl) during co-transformation resulted in an increase in frequency for double recombination from 16 to $45 \%$ (Supplementary Figure S3). This suggests that the proportion of gRNA and dDNA of multiple genes requires optimization to achieve a high frequency of simultaneous multirecombination.

This study demonstrates precise editing of T. reesei genome through the repair of Cas9-induced DSBs in an NHEJ or homology-recombination method. However, unwanted insertion and deletion mutations were observed at off-target sites, which share high sequence similarity with the on-target site in other organisms [31-33]. We tried to avoid the selection of target sites sharing high sequence similarity with other sites in the $T$. reesei genome in this study. However, this is only one approach [34-36] used to reduce rather than eliminate off-target sites. Thus, it is possible that off-target might also occur in our $T$. reesei CRISPR/Cas9 system. It is an important issue to eliminate the off-target effect in the CRISPR/Cas9 system as well as the other genome-editing tools [37, 38]; specific experiments to test the offtarget effect in $T$. reesei CRISPR/Cas9 system should be designed in a future study.

In this study, the primary applications of the CRISPR/Cas9 system have been demonstrated as model genome-engineering methods in filamentous fungi, which can accelerate research on the functional genomics of filamentous fungi. Numerous applications of the CRISPR/Cas9 system have been developed in other organisms within the past year. Similarly, more CRISPR/Cas9-mediated systems could be examined in filamentous fungi. For example, a CRISPR/Cas9-mediated large fragment deletion would facilitate genetic modification of filamentous fungal chassis [31]. A catalytically deactivated version of Cas9 can be used for genome-scale CRISPR-mediated control of gene repression and activation [10]. However, until now gRNAs could not be synthesized in vivo easily in $T$. reesei, which may limit its application fields. It is important to allow gRNA to be synthesized in vivo by identifying effective RNA polymerase-III-based promoters in $T$. reesei.

\section{Materials and Methods}

\section{Strains and plasmids}

E. coli $\mathrm{DH} 5 \alpha$ served as the cloning host (Novagen, Gibbstown, NJ, USA). A. tumefaciens AGL1 was used to transform the Cas 9 gene to T. reesei strains [39]. The T. reesei strain Qm6a (13631) and Rut-C30 (56765) purchased from American Type Culture Collection (ATCC, Manassas, VA, USA), were used as the hosts for Cas9 expression. A T-DNA binary vector, $\mathrm{pDHt} /$ sk, containing the hph coding for hygromycin B phosphotransferase (under control of the $A$. nidulans trpC promoter and terminator) was used to construct the transformation vectors. pMD-18T vector (Takara, Dalian, China) was used to maintain gRNA templates and dDNAs in DH5 $\alpha$.

\section{Expression of Cas9 in $T$. reesei}

The Cas9 gene with a SV40 nuclear localization signal was codon-optimized for expression in $T$. reesei (toCas9, Supplementary Data S1). To express to Cas 9 efficiently, a constitutive promoter Ppdc and an inducible promoter Pcbh1 were used to express to Cas9 in Qm6a and Rut-C30, respectively. The $e G F P$ gene was fused to to $\operatorname{Cas} 9$ as a tag. All the cassettes with Tpdc as terminator fused into $\mathrm{XbaI}$ site of the linearized pDHt/sk using the ClonExpress MultiS One Step Cloning Kit (Vazyme, Nanjing, China) to generate four vectors, $\mathrm{pDH} / \mathrm{sk}-\mathrm{Ppdc}-$ toCas -Tpdc (pDHt/sk-PC), $\mathrm{pDHt} / \mathrm{sk}-\mathrm{Ppdc}$ toCas9-eGFP-Tpdc (pDHt/sk-PE), pDHt/sk-Pcbh1-toCas9Tpdc (pDHt/sk-CC), and pDHt/sk-Pcbh1-toCas9-eGFP-Tpdc (pDHt/sk-CE) (Table 1). We also constructed vectors only bearing the $e G F P$ gene (pDHt/sk-Ppdc-eGFP-Tpdc and $\mathrm{pDHt} /$ sk-Pcbh1-eGFP-Tpdc) as controls. All of the expression vectors were transformed into the recipient $T$. reesei using Agrobacteriummediated fungal transformation [40]. Transformants were selected using hygromycin $\mathrm{B}\left(10 \mu \mathrm{g} \mathrm{ml}^{-1}\right)$ and cefotaxime $(300 \mu \mathrm{M})$ on potato dextrose agar. Corresponding to the expression vector, the transformants derived from Qm6a (with constitutive promoter) were named 6a-pc, 6a-pe, and 6a-gfp and ones from Rut-C30 (with inducible promoter) were named C30-cc, C30-ce, and C30gfp. Each positive transformant was used to create monoconidial cultures for genetic stability test

The positive transformants with eGFP tag under Ppdc and Pcbh1 were grown on MM [40] with 2\% glucose and 1\% Avicel as carbon resources, respectively. The mycelium was collected by filtration and used to extract intracellular proteins after grinding in liquid nitrogen. Fusion protein production was examined by SDS-polyacrylamide gel electrophoresis and western blots using an anti-GFP antibody (Genscript, Nanjing, China).

\section{Protoplast transformation of $g R N A$}

The gRNA cassette including synthetic gRNA sequence and target DNA of the ura5 gene (5'-GGCGAGGGCGGCAAC ATCGT $c g g-3^{\prime}$, PAM is shown in italics) was controlled by T7 
promoter (Supplementary Data S1). This cassette was ligated into pMD-18T and maintained in DH5 $\alpha$ as template for the following transcription. Before transformation, the cassette was transcribed into RNA in vitro using the MEGAscript T7 Kit (Ambion, Austin, TX, USA). The generated RNA fragments $(\geqslant 10 \mu \mathrm{g})$ were transformed into the Cas9-expressing chassis (6apc and $\mathrm{C} 30-\mathrm{cc}$, respectively) using a modified polyethylene glycol-mediated protoplast transformation procedure [41]. To express Cas 9 as efficiently as possible, different carbon resources were used in screening MM plates corresponding to the promoters as mentioned in the previous section. Finally, the nonsense mutants of URA5 were selected in these modified MM plates including the $1.5 \mathrm{mg} \mathrm{ml}^{-1}$ 5-FOA (Sangon, Shanghai, China) and $10 \mathrm{~mm}$ Uridine (Sangon). The genomic DNAs of selected transformants were extracted using the FastPrep instrument (MP Biomedicals, Santa Ana, CA, USA) with a standard protocol and used for the amplification of the gene ura5. Sequence analysis of the partial ura5 from each transformant was carried out by BioSune (Shanghai, China).

\section{Co-transformation of $g R N A$ with donor $D N A$}

The generated uridine-dependent Cas 9 expression chassis (AR3) derived from $\mathrm{C} 30$-cc was used as a host to construct an efficient CRISPR-Cas-directed homologous recombination system. Similar to URA5 mutagenesis, the CRISPR-Cas target site (5'-GGATGCCGAAATCATGGCCGtgg-3', PAM is shown in italics) (Supplementary Data S1) was located in lae1 [13]. The dDNA (dDNA-lae1) containing the 5' and 3' flanking sequences of lael and the selectable marker cassette (the ura5 gene from $P$. oxalicum controlled by the Pgpda promoter and Ttrpc terminator, Pgpda-poura5-Ttrpc) (Supplementary Data S1) was generated by overlapping PCR using Q5 (NEB, Beijing, China) and ligated into the pMD-18T vector. The generated vector was propagated in DH5 $\alpha$ and purified using the Plasmid Midi Kit (Qiagen, Hilden, Germany). Accompanying the RNA fragments including gRNA and the target sequence of lae1, dDNA was introduced into T. reese $i$ using the protoplast transformation procedure. The transformants were selected using MM plates with 1\% lactose as an inducer of Cas9.

Construction of $d D N A s$ with different homology arm sizes To examine the effect of homology arm length on targeted gene replacements in $T$. reesei, dDNAs were constructed using homology arms at various sizes. In addition to dDNA-lae1, Pgpda-poura5-Ttrpc was used as a selectable marker. Besides the $1-\mathrm{kb}$ flanking sequences (dDNA-lae1), the 0.2-, 0.4-, 0.6-, and $0.8-\mathrm{kb}$ flanking sequences of lael were fused to the selectable marker. The generated dDNAs were named dDNAlae1-0.2k, dDNA-lae1-0.4k, dDNA-lae1-0.6k, and dDNA-lae1$0.8 \mathrm{k}$ (Supplementary Data $\mathrm{S} 1$ ) and were ligated into the pMD-18T vector. The vector was propagated in DH5 $\alpha$ and purified using the Plasmid Midi Kit (Qiagen). The cotransformation procedure was similar to that described above.

\section{Co-transformation of multiple fragments}

Multiple genome modification was also performed in the uridine-dependent Cas9-expressing chassis AR3 through co-transformation of two sets of gRNA and dDNA. In addition to the locus of lae1, another target site (5'-GGCTTCTGTCAA GACAACCTtgg-3', PAM is shown in italics) (Supplementary Data S1) was located in the gene encoding VIB1, a putative link between glucose signaling and carbon catabolite repression [15]. The dDNA (dDNA-vibl) containing the 5'- and $3^{\prime}$ flanking sequences of vibl and the selectable marker cassette (Pgpdapoura5-Ttrpc) (Supplementary Data S1) was constructed as similarly to dDNA-lae1. In the triple-loci assay, clr2 (5'-GGA CGAGGAACGAAGAGACCggg-3', PAM is shown in italics) (Supplementary Data S1) was used as the third target, which was a conserved and essential transcription factor for cellulase gene expression in ascomycete fungi [16]. The dDNA (dDNA-clr2) containing the $5^{\prime}$ and $3^{\prime}$ flanking sequences of clr2 and the selectable marker cassette (Pgpda-poura5-Ttrpc) (Supplementary Data S1) was constructed similarly to dDNA-lae1. After quantitating to the same molar concentration, two or three sets of gRNA and dDNA were introduced into $T$. reese $i$ using the protoplast transformation procedure. The transformants were also selected using the MM plate with an inducer.

\section{Conflict of Interest}

The authors declare no conflict of interest.

\section{Acknowledgements}

This work was financially supported by National Basic Research Program of China (973: 2011CB707403), High-tech Research and Development Program of China (863: 2013AA102806) and the National Natural Science Foundation of China (31300073, 31470201).

\section{References}

1 Punt PJ, van Biezen N, Conesa A, Albers A, Mangnus J, van den Hondel C. Filamentous fungi as cell factories for heterologous protein production. Trends Biotechnol 2002; 20: $200-206$.

2 Jiang D, Zhu W, Wang Y, Sun C, Zhang KQ, Yang J. Molecular tools for functional genomics in filamentous fungi: recent advances and new strategies. Biotechnol $A d v$ 2013; 31: 1562-1574.

3 Burgess DJ. Technology: a CRISPR genome-editing tool. Nat Rev Genet 2013; 14: 80.

4 Carroll D. Genome engineering with zinc-finger nucleases. Genetics 2011; 188: 773-782.

5 Cermak T, Doyle EL, Christian M et al. Efficient design and assembly of custom TALEN and other TAL effector-based constructs for DNA targeting. Nucleic Acids Res 2011; 39: e82.

6 Cong L, Ran FA, Cox D et al. Multiplex genome engineering using CRISPR/Cas systems. Science 2013; 339: 819-823.

7 DiCarlo JE, Norville JE, Mali P, Rios X, Aach J, Church GM. Genome engineering in Saccharomyces cerevisiae 
using CRISPR-Cas systems. Nucleic Acids Res 2013; 41: 4336- 4343.

8 Li J, Wang J, Wang S, Xing M, Yu S, Liu G. Achieving efficient protein expression in Trichoderma reesei by using strong constitutive promoters. Microb Cell Fact 2012; 11: 84.

9 Ilmén M, Onnela ML, Klemsdal S, Keränen S, Penttilä M. Functional analysis of the cellobiohydrolase I promoter of the filamentous fungus Trichoderma reesei. Mol Gen Genet 1996; 253: 303-314.

10 Gilbert LA, Larson MH, Morsut L et al. CRISPRmediated modular RNA-guided regulation of transcription in eukaryotes. Cell 2013; 154: 442-451.

11 Wang Y, Li Z, Xu J et al. The CRISPR/Cas system mediates efficient genome engineering in Bombyx mori. Cell Res 2013; 23: 1414-1416

12 Liu G, Zhang L, Wei X et al. Genomic and secretomic analyses reveal unique features of the lignocellulolytic enzyme system of Penicillium decumbens. PLoS One 2013; 8: e55185.

13 Seiboth B, Karimi RA, Phatale PA et al. The putative protein methyltransferase LAE1 controls cellulase gene expression in Trichoderma reesei. Mol Microbiol 2012; 84: $1150-1164$.

14 Meyer V, Arentshorst M, El-Ghezal A et al. Highly efficient gene targeting in the Aspergillus niger kusA mutant. $J$ Biotechnol 2007; 128: 770-775.

15 Xiong Y, Sun J, Glass NL. VIB1, a link between glucose signaling and carbon catabolite repression, is essential for plant cell wall degradation by Neurospora crassa. PLoS Genet 2014; 10: e1004500.

16 Coradetti ST, Craig JP, Xiong Y, Shock T, Tian CG, Glass NL. Conserved and essential transcription factors for cellulase gene expression in ascomycete fungi. Proc Natl Acad Sci USA 2012; 109: 7397-7402.

17 Ballance DJ, Buxton FP, Turner G. Transformation of Aspergillus nidulans by the orotidine-5'-phosphate decarboxylase gene of Neurospora crassa. Biochem Biophys Res Commun 1983; 112: 284-289.

18 Tilburn J, Scazzocchio C, Taylor GG, Zabicky-Zissman $\mathrm{JH}$, Lockington RA, Davies RW. Transformation by integration in Aspergillus nidulans. Gene 1983; 26: 205-221.

19 Penttilä M, Nevalainen H, Rättö M, Salminen E, Knowles J. A versatile transformation system for the cellulolytic filamentous fungus Trichoderma reesei. Gene 1987; 61: $155-164$.

20 Herzog RW, Daniell H, Singh NK, Lemke PA. A comparative study on the transformation of Aspergillus nidulans by microprojectile bombardment of conidia and a more conventional procedure using protoplasts treated with polyethyleneglycol. Appl Microbiol Biot 1996; 45: 333-337.

21 Frandsen RJN. A guide to binary vectors and strategies for targeted genome modification in fungi using Agrobacterium tumefaciens-mediated transformation. J Microbiol Methods 2011; 87: 247-262.

22 Kanaar R, Hoeijmakers JH, van Gent DC. Molecular mechanisms of DNA double strand break repair. Trends Cell Biol 1998; 8: 483-489.
23 Weld RJ, Plummer KM, Carpenter MA, Ridgway HJ. Approaches to functional genomics in filamentous fungi. Cell Res 2006; 16: 31-44.

24 van Attikum H, Bundock P, Hooykaas PJ. Nonhomologous end-joining proteins are required for Agrobacterium T-DNA integration. EMBO J 2001; 20: 6550-6558.

25 Zhang G, Hartl L, Schuster A et al. Gene targeting in a nonhomologous end joining deficient Hypocrea jecorina. J Biotechnol 2009; 139: 146-151.

26 Krappmann S, Sasse C, Braus GH. Gene targeting in Aspergillus fumigatus by homologous recombination is facilitated in a nonhomologous end-joining-deficient genetic background. Eukaryot Cell 2006; 5: 212-215.

27 Fu K, Liu L, Fan L, Liu T, Chen J. Accumulation of copper in Trichoderma reesei transformants, constructed with the modified Agrobacterium tumefaciens-mediated transformation technique. Biotechnol Lett 2010; 32: 1815-1820.

28 Colot HV, Park G, Turner GE et al. A high-throughput gene knockout procedure for Neurospora reveals functions for multiple transcription factors. Proc Natl Acad Sci USA 2006; 103: 10352-10357.

29 Park G, Colot HV, Collopy PD et al. High-throughput production of gene replacement mutants in Neurospora crassa. Methods Mol Biol 2011; 722: 179-189.

$30 \mathrm{Bi} \mathrm{X}$. Heterochromatin structure: Lessons from the budding yeast. IUBMB Life 2014; 66: 657-666.

31 Essletzbichler P, Konopka T, Santoro F et al. Megabasescale deletion using CRISPR/Cas9 to generate a fully haploid human cell line. Genome Res 2014; 24: 2059-2065.

32 Hruscha A, Krawitz P, Rechenberg A et al. Efficient CRISPR/Cas9 genome editing with low off-target effects in zebrafish. Development 2013; 140: 4982-4987.

33 Veres A, Gosis BS, Ding QR et al. Low Incidence of offtarget mutations in individual CRISPR-Cas9 and TALEN targeted human stem cell clones detected by whole-genome sequencing. Cell Stem Cell 2014; 15: 254-254.

34 Ran FA, Hsu PD, Lin CY et al. Double nicking by RNAguided CRISPR Cas9 for enhanced genome editing specificity. Cell 2013; 155: 479-480.

35 Ansai S, Kinoshita M. Targeted mutagenesis using CRISPR/Cas system in medaka. Biol Open 2014; 3: 362-371.

36 Fu Y, Sander JD, Reyon D, Cascio VM, Joung JK. Improving CRISPR-Cas nuclease specificity using truncated guide RNAs. Nat Biotechnol 2014; 32: 279-284.

37 Miller GL. Use of dinitrosalicylic acid reagent for determination of reducing sugar. Anal Chem 1959; 31: 426-428.

38 Grau J, Boch J, Posch S. TALENoffer: genome-wide TALEN off-target prediction. Bioinformatics 2013; 29: 2931-2932.

39 Ram AFJ, Michielse CB, Hooykaas PJJ, van den Hondel CAMJJ. Agrobacterium-mediated transformation of the filamentous fungus Aspergillus awamori. Nat Protoc 2008; 3: 1671-1678.

40 Zou G, Shi S, Jiang Y et al. Construction of a cellulase hyper-expression system in Trichoderma reesei by 
promoter and enzyme engineering. Microb Cell Fact 2012; 11: 21 .

41 Liu T, Wang T, Li X, Liu X. Improved heterologous gene expression in Trichoderma reesei by cellobiohydrolase I gene $(c b h l)$ promoter optimization. Acta Bioch Bioph Sin 2008; 40: 158-165.

(Supplementary Information is linked to the online version of the paper on the Cell Discovery website.) (c) (i) (2) $\Theta$ This work is licensed under a Creative Commons Attribution-NonCommercial-NoDerivs 4.0 International License. The images or other third party material in this article are included in the article's Creative Commons license, unless indicated otherwise in the credit line; if the material is not included under the Creative Commons license, users will need to obtain permission from the license holder to reproduce the material. To view a copy of this license, visit http:// creativecommons.org/licenses/by-nc-nd/4.0/ 